\title{
Temporal Evolution of the Solar-Wind Electron Core Density at Solar Minimum by Correlating SWEA Measurements from STEREO A and B
}

\author{
A. Opitz · J.-A. Sauvaud • A. Fedorov • P. Wurz • J.G. Luhmann · B. Lavraud • \\ C.T. Russell · P. Kellogg • C. Briand · P. Henri · D.M. Malaspina · P. Louarn • \\ D.W. Curtis · E. Penou · R. Karrer • A.B. Galvin · D.E. Larson · I. Dandouras • \\ P. Schroeder
}

Received: 1 October 2009 / Accepted: 12 July 2010 / Published online: 28 August 2010

(C) The Author(s) 2010. This article is published with open access at Springerlink.com

\begin{abstract}
The twin STEREO spacecraft provide a unique tool to study the temporal evolution of the solar-wind properties in the ecliptic since their longitudinal separation increases with time. We derive the characteristic temporal variations at $\sim 1$ AU between two different plasma parcels ejected from the same solar source by excluding the spatial variations from our datasets. As part of the onboard IMPACT instrument suite, the SWEA electron experiment provides the solar-wind electron core density at two different heliospheric vantage points. We analyze these density datasets between March and August 2007 and find typical solar minimum conditions. After adjusting for the theoretical time lag between the two spacecraft, we compare the two density datasets. We find that their correlation decreases
\end{abstract}

\footnotetext{
A. Opitz $(\bowtie) \cdot$ J.-A. Sauvaud · A. Fedorov · B. Lavraud · P. Louarn · E. Penou · I. Dandouras Centre d'Etude Spatiale des Rayonnements (CNRS-UPS), University of Toulouse, Toulouse, France e-mail: opitz@cesr.fr

P. Wurz · R. Karrer

Department of Space Science and Planetology, Physics Institute, University of Bern, Bern, Switzerland

J.G. Luhmann · D.W. Curtis · D.E. Larson · P. Schroeder

Space Sciences Laboratory, University of California, Berkeley, USA

C.T. Russell

Institute of Geophysics and Planetary Physics, University of California, Los Angeles, USA

P. Kellogg

Department of Physics and Astronomy, University of Minnesota, Minneapolis, USA

C. Briand · P. Henri

LESIA, Observatoire de Paris, CNRS, Université Pierre et Marie Curie, Université Paris-Diderot, Meudon, France

D.M. Malaspina

Laboratory for Atmospheric and Space Physics, University of Colorado, Boulder, CO, USA
}

A.B. Galvin

Space Science Center, University of New Hampshire, Durham, USA 
as the time difference increases between two ejections. The correlation coefficient is about 0.80 for a time lag of a half day and 0.65 for two days. These correlation coefficients from the electron core density are somewhat lower than the ones from the proton bulk velocity obtained in an earlier study, though they are still high enough to consider the solar wind as persistent after two days. These quantitative results reflect the variability of the solar-wind properties in space and time, and they might serve as input for solar-wind models.

\section{Introduction}

The temporal evolution of the solar wind was recently studied by Opitz et al. (2009) analyzing the solar-wind proton bulk velocity. The present paper extends this work by analyzing the density of solar-wind electrons. The observed solar-wind electron velocity distributions are composed of three different components: the thermal core, the suprathermal halo, and the strahl aligned with the interplanetary magnetic field (Feldman et al., 1975; Pilipp et al., 1987; McComas et al., 1992; Maksimovic et al., 2005; Marsch, 2006; etc.). The bulk of the electrons $(\sim 95 \%)$ are at low energies in the core, which is a cold $\left(\sim 10^{5} \mathrm{~K}\right)$ and isotropic (due to Coulomb collisions) population. The halo population represents together with the antisunward drifting strahl the remaining portion of the total electron number density. Here we correlate core density measurements between two different spacecraft after accounting for spatial effects in order to derive the temporal evolution of the solar-wind electron core population.

The Solar Terrestrial Relations Observatory (STEREO) mission (Kaiser et al., 2008) is an excellent opportunity to examine the temporal evolution of the solar wind by two spacecraft with nearly identical instrumentation. The STEREO A (Ahead) and B (Behind) spacecraft are positioned at two different vantage points in the ecliptic and their longitudinal separation increases by $\sim 45^{\circ}$ per year, thus enabling two-point correlation analysis from two widely spaced probes. We analyze in situ electron data measured during solar minimum between March and August 2007. In this time period the longitudinal spacecraft separation is monotonically increasing from $1.2^{\circ}$ to $28.2^{\circ}$, hence the time difference between two detected ejections from the same solar source is also increasing from 0.09 up to 2.14 days.

\section{SWEA Dataset}

The In situ Measurements of Particles and CME Transients (IMPACT) investigation (Luhmann et al., 2008) on the STEREO mission provides multipoint solar-wind electron, interplanetary magnetic field, and solar energetic particle measurements. As part of this complex instrument package the Solar Wind Electron Analyzer (SWEA) experiment (Sauvaud et al., 2008) provides in situ electron measurements at both STEREO spacecraft in the energy range of $1-2000 \mathrm{eV}$.

SWEA is mounted at the end of the IMPACT boom on the antisunward side of the STEREO spacecraft in order to avoid direct sunlight entering into our particle detector. SWEA consists of an electrostatic analyzer, an MCP detector, and deflector plates at the aperture. As allowed by the deflector plates, the instrument provides measurements over a substantial part of the unit sphere with viewing solid angle of $360^{\circ} \times 120^{\circ}$. From these measurements we derive the electron core and halo parameters, we produce the pitch angle distributions, and calculate the heat flux with 30-s time resolution (http://stereo.cesr.fr). At energies lower than $\sim 60 \mathrm{eV}$, the transmission of the SWEA analyzer is very weak due to 
a charging effect on the tophat itself. In order to compute the solar-wind core density, we had to take into account the flux of secondary electrons created in the analyzer to infer the flux of incoming solar-wind electrons. More details are given by Fedorov et al. (2010). Our density results were cross-calibrated with measurements from several other experiments; we used SWAVES (Bougeret et al., 2008) electron density results to define the absolute value. Note that the SWEA core density data is sufficiently clean so that despiking was not needed before the statistical analysis. At low densities the uncertainty in the density data increases, which might be due to photoelectrons that we neglected during data processing.

The top row of Figure 1 (both Figures 1(a) and (b)) shows the SWEA solar-wind electron core density measurements. The range of density variations is generally small, except when recurrent huge enhancements are observed. These enhancements are related to compression regions that are formed where fast and slow solar-wind streams interact. The measured density pattern is typical for in-ecliptic solar-wind conditions during solar minimum.

\section{Analysis, Results and Discussion}

In order to derive the temporal evolution of the solar-wind electron core density we perform regression and correlation analysis of the SWEA measurements after adjusting for the theoretical time lag. The applied method is described in detail by Opitz et al. (2009), where they calculate the time lag between the STEREO A and B spacecraft by separating the effect of the radial and longitudinal spacecraft separation. The time lag from the longitudinal separation is calculated from the solar rotation rate. The time lag from the radial separation is calculated with the solar-wind proton bulk velocity measured by Plasma and Suprathermal Ion Composition experiment (PLASTIC; Galvin et al., 2008). Since the density of the electrons tracks the density of the ions which move radially outward at the solar-wind bulk velocity, we assume that the fluctuations in density of the solar-wind core electrons propagate radially outward with the proton bulk velocity as needed to fulfill locally the quasineutrality. We also assume that this velocity remains constant, which is valid on such distances like the small radial separation of the two STEREO spacecraft. The latitudinal effects are neglected since both STEREO spacecraft are nearly in the ecliptic plane. In case of occasional large latitudinal gradients, omission of the latitudinal spacecraft separation might introduce a mismatch even at small separations.

After shifting the B dataset by the obtained theoretical time lag (Figure 1, second row), the agreement between the A and B datasets is clearly improved (compare the first and second rows of Figure 1(b) in particular). We search for A and B data pairs, which allows to perform correlation analysis between the two density datasets (Figure 1, third row). Note these correlations are performed using a time window of one-third of a Carrington rotation. The corresponding regression plots are shown in the bottom row of Figure 1.

These solar-wind electron core density results are similar to those obtained for the solarwind bulk velocity by Opitz et al. (2009). The major correlation drops for the proton velocity are visible for the electron density as well. We note in particular the effects of the May 2007 $(\sim$ DOY 142$)$ coronal mass ejection (CME) and an exceptionally fast temporal evolution of the solar wind in August 2007 (DOY 227-232). The latter event was immediately followed by a latitudinal effect on the dataset: there was a large latitudinal gradient of the bulk velocity due to the proximity of a coronal hole, hence the latitudinal spacecraft separation was not negligible anymore. We must admit that the density analysis resulted in surprisingly low correlation coefficients in April 2007 (DOY 95-118), which we cannot explain at the moment.

In order to study different scales in the solar wind we use different time windows in Figure 2. A time window of one Carrington rotation (CR) delivers information about the 

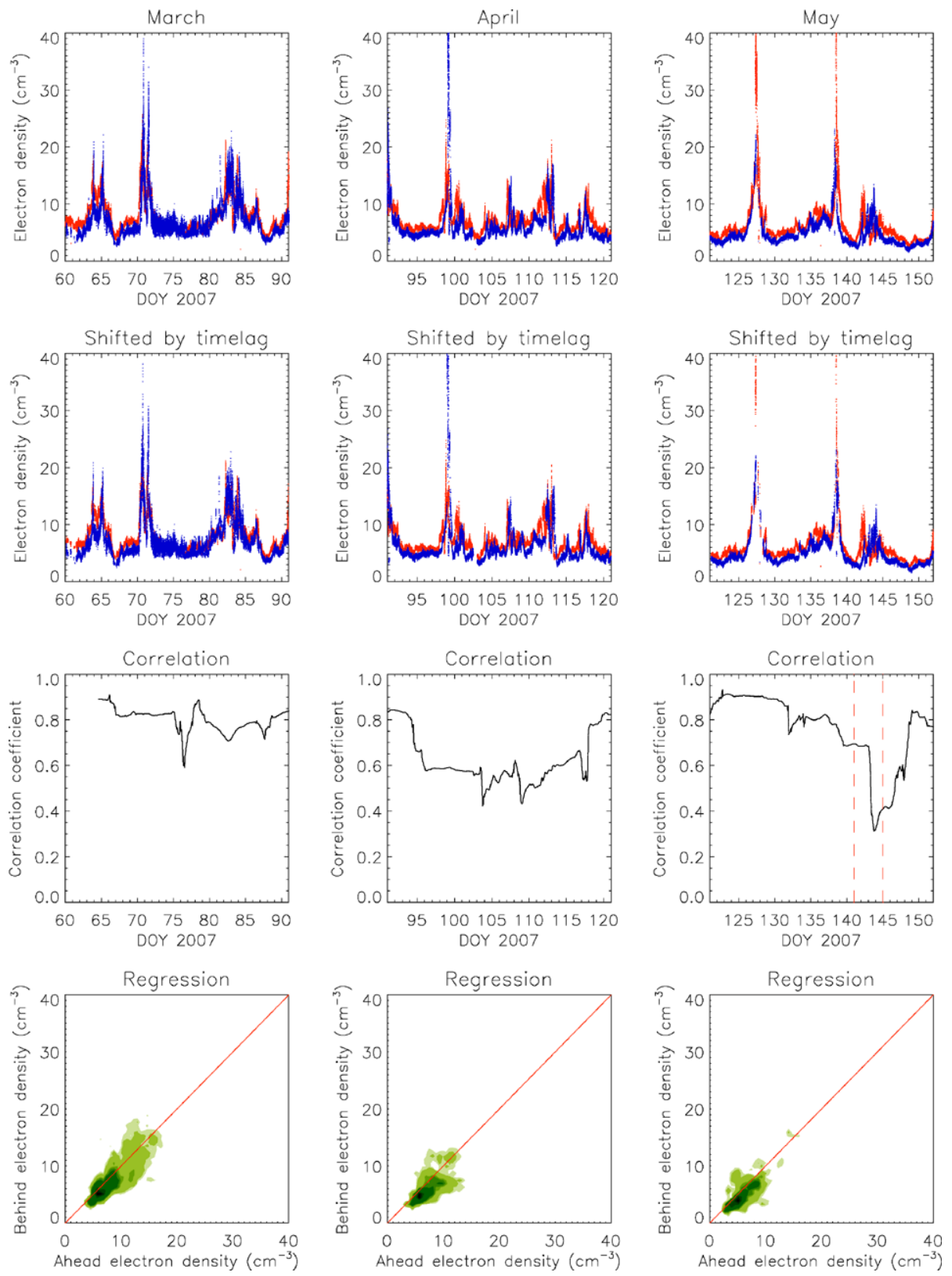

Figure 1a Data plots for March-May 2007. Top row: calibrated half-minute STEREO A (red) and B (blue) SWEA solar-wind electron core density data. Second row: The same data as in the top row, but the B dataset is shifted by the radial and longitudinal time lag. Third row: Correlation between the A and time-shifted B electron core density data over one-third of a Carrington rotation time period (CR/3). Fourth row: Regression plot of the A and time-shifted B electron core density data. On the correlation plot of May, vertical red dashed lines show the time period when the plasma parameters were affected by a CME. 

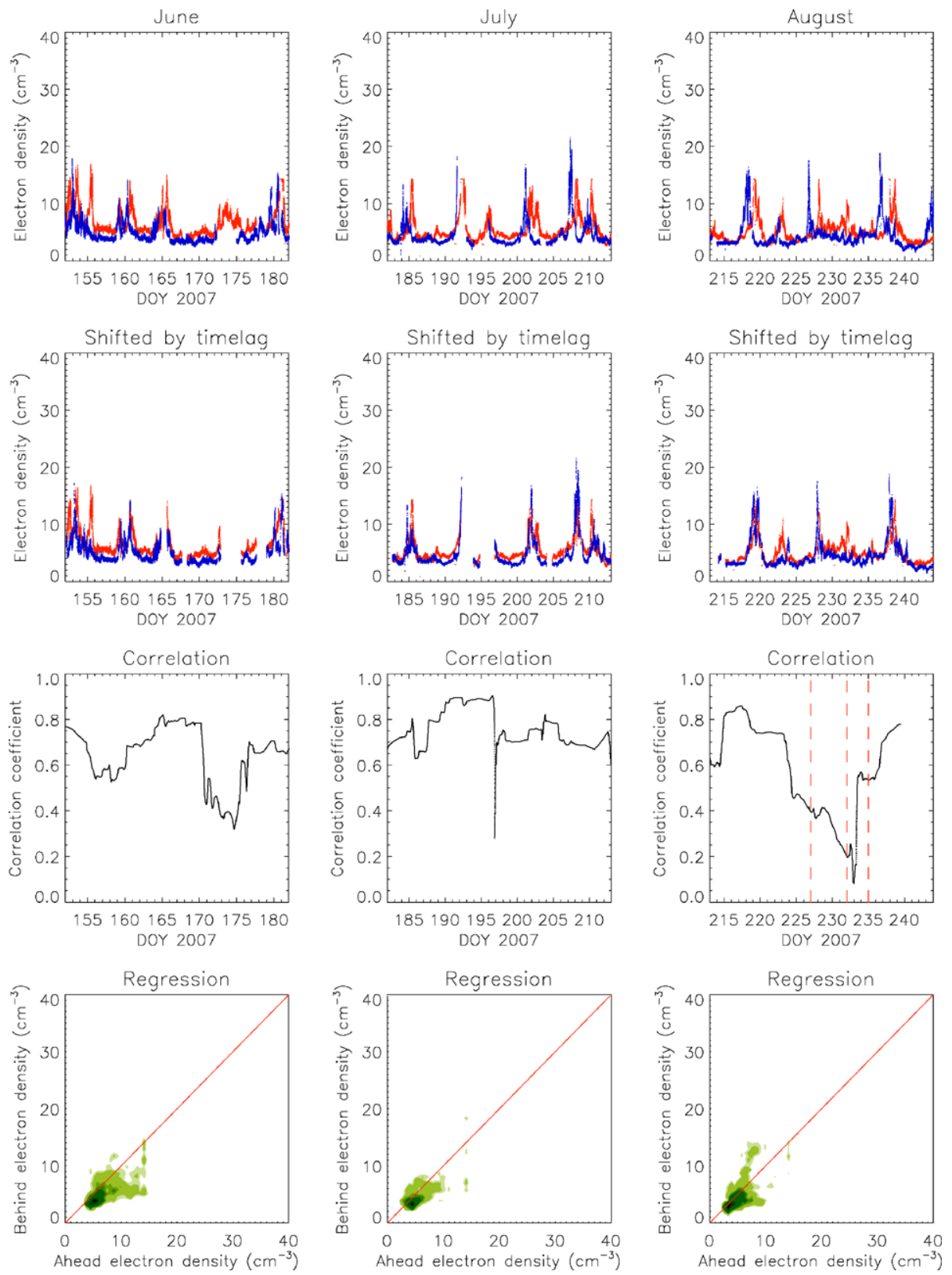

Figure 1b Data plots for June-August 2007. Top row: calibrated half-minute STEREO A (red) and B (blue) SWEA solar-wind electron core density data. Second row: The same data as in the top row, but the B dataset is shifted by the radial and longitudinal time lag. Third row: Correlation between the A and time-shifted B electron core density data over one-third of a Carrington rotation time period (CR/3). Fourth row: Regression plot of the A and time-shifted B electron core density data. On the correlation plot of August, vertical red dashed lines show the time period when there was a fast temporal evolution (DOY 227-232) and immediately after that a latitudinal effect (DOY 232-235). 

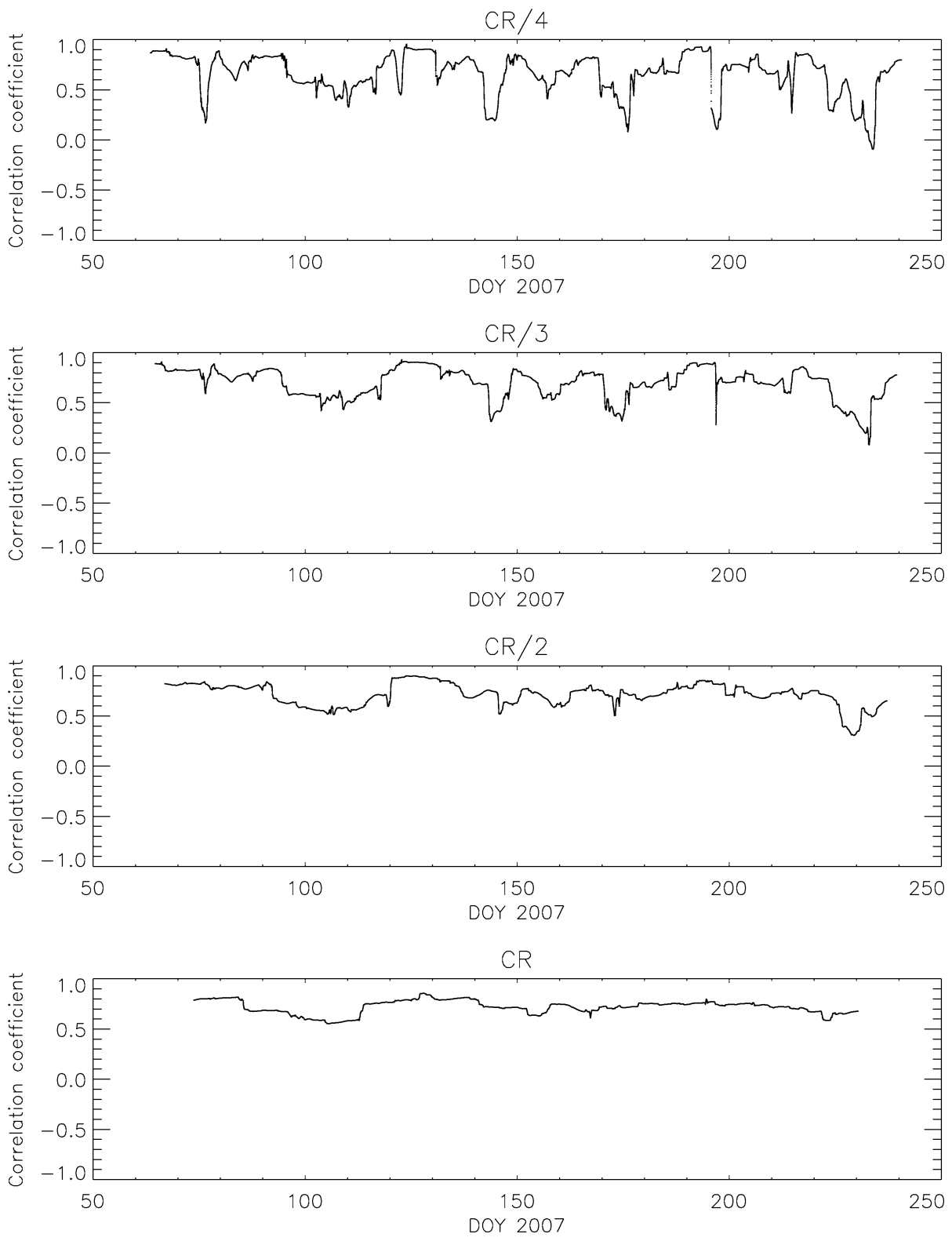

Figure 2 Correlation over different time windows between STEREO A and time-shifted B SWEA solar-wind electron core density data for March-August 2007 with half-minute resolution. Top panel: One-quarter Carrington rotation (CR/4). Second panel: One-third Carrington rotation (CR/3). Third panel: One-half Carrington rotation $(\mathrm{CR} / 2)$. Fourth panel: One Carrington rotation (CR). The correlation coefficient is always plotted in the center of the applied time period; for instance, the correlation coefficient on DOY 100 in the top panel $(\mathrm{CR} / 4)$ was calculated from measurements between DOY $100-(\mathrm{CR} / 4) / 2$ and DOY $100+(\mathrm{CR} / 4) / 2$. 
Figure 3 STEREO A and B

SWEA solar-wind electron core density correlation results calculated over a time window of one Carrington rotation as a function of the time lag from the longitudinal separation of the two STEREO spacecraft.

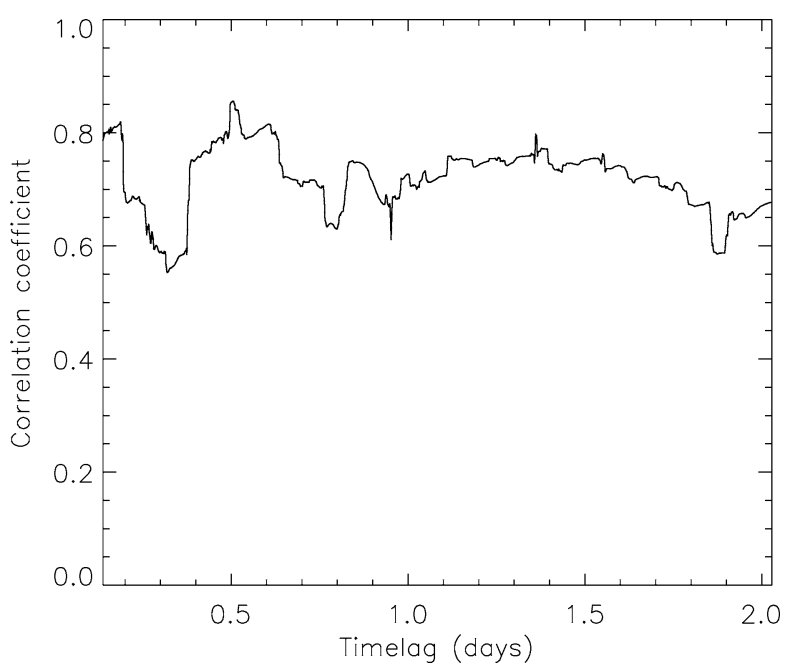

temporal evolution of the whole Sun. One-half of a Carrington rotation $(\mathrm{CR} / 2)$ reflects solar wind emerged just from half of the Sun. One-third of a Carrington rotation $(\mathrm{CR} / 3$; same as in Figure 1) was chosen because there were about three high density features (in agreement with the three fast solar-wind features) per Carrington rotation in the investigated dataset. One-fourth of a Carrington rotation $(\mathrm{CR} / 4)$ is about one-week and allows the study of smaller scale solar-wind features. For increasing length of this time window, which corresponds to larger spatial scales, the correlation becomes smoother since the temporal evolution of smaller scales is averaged. This was also found from ion data by Richardson, Dashevskiy, and Paularena (1998). The correlation coefficient is decreased if small-scale structures are detected by one spacecraft and not by the other one. Two other reasons for the correlation coefficient decrease as the correlation time window is decreased was given by Coplan et al. (2001): the lower correlation coefficients for higher time-resolution data are due to the larger random component and fewer plasma parameter changes. Note that the influence of the data variability on the correlation coefficient can explain the spikiness of the top panel of Figure 2. Here the one-fourth Carrington rotation time period is shorter than the occurrence rate of the density enhancements. As a result, the applied time window might include an enhancement or not, hence the data variability can be very different in accord with the correlation coefficient.

The temporal evolution of the density of solar-wind core electrons is well described in Figure 3 by plotting the correlation coefficient as a function of the time lag from the longitudinal separation. It describes the evolution of the solar wind between two ejections from the same solar source, but at different times. As this time difference increases, the solar-wind density differs more since it had more time to evolve. As long as the correlation coefficient is about 0.80 for a time lag of a half day, it decreases to $\sim 0.65$ for two days time lag. Even the latter coefficient allows us to consider the solar wind as persistent.

\section{Conclusions}

The temporal evolution of the solar wind was investigated by correlating the STEREO A and B SWEA solar wind core electron density measurements. We found that the adjustment for 
the theoretical time lag results in improved agreement between the A and B datasets. The correlations are good and only show a slightly decreasing tendency as the time increases between two plasma parcel ejections from the same solar source. The correlation coefficient is on average about 0.80 for a time lag of a half day and $\sim 0.65$ for two days. The large drops in the correlation coefficient are due to deviations from the nominal conditions, they indicate interesting events, such as CMEs or large latitudinal gradients. Our solar-wind electron core density results agree qualitatively with the proton bulk velocity results by Opitz et al. (2009). The analysis of the density delivers lower correlations than the bulk velocity, which was also found by Richardson, Dashevskiy, and Paularena (1998). This might be either due to more random components in the dataset, or due to more local influences on density than on velocity, or due to less variability in the density than the velocity dataset. Most probably the reason is a combination of all these effects. Note also that the decreasing tendency is flatter for density. In order to say more, these two studies should be extended. When the STEREO longitudinal spacecraft separation will increase up to $180^{\circ}$, we will derive the temporal evolution of the solar-wind properties for any time lag.

This study reflects quantitatively the solar-wind variability in space and time. Our results can be used as input in solar-wind models for the quiet Sun, as long as the deviations from the good correlations are indicators of solar events or exceptional conditions. The finding that the solar wind is persistent even after two days (equivalent to $\sim 30^{\circ}$ solar rotation) is important to validate the extrapolation of solar-wind properties to other heliospheric longitudes. Such predictions were performed from STEREO measurements by Opitz et al. (2010), where they predicted the solar-wind proton bulk velocity at Venus, Earth and Mars.

Acknowledgements The authors acknowledge contribution from the entire STEREO group, especially the IMPACT and PLASTIC experiment teams. Another thank goes to the colleagues working with SWAVES for cross-calibration. We thank for discussions with Milan Maksimovic, Alexis Rouillard, David J. McComas, Teresa Nieves-Chinchilla, Ioannis Zouganelis and Karim Meziane. This work was supported by a CNES Grant for CESR (CNRS-UPS).

Open Access This article is distributed under the terms of the Creative Commons Attribution Noncommercial License which permits any noncommercial use, distribution, and reproduction in any medium, provided the original author(s) and source are credited.

\section{References}

Bougeret, J.L., Goetz, K., Kaiser, M.L., Bale, S.D., Kellogg, P.J., Maksimovic, M., Monge, N., Monson, S.J., Astier, P.L., Davy, S., et al.: 2008, Space Sci. Rev. 136, 487.

Coplan, M.A., Ipavich, F., King, J., Ogilvie, K.W., Roberts, D.A., Lazarus, A.J.: 2001, J. Geophys. Res. 106(A9), 18615.

Fedorov, A., Opitz, A., Sauvaud, J.-A., Luhmann, J., Curtis, D.W., Larson, D.E.: 2010, Space Sci. Rev., in press.

Feldman, W.C., Asbridge, J.R., Bame, S.J., Montgomery, M.D., Gary, S.P.: 1975, J. Geophys. Res. 80(31), 4181.

Galvin, A.B., Kistler, L.M., Popecki, M., Farrugia, C.J., Simunac, K.D.C., Ellis, L., Ellis, S., Gaidos, J.A., Granoff, M., Heirtzler, D., et al.: 2008, Space Sci. Rev. 136, 437.

Kaiser, M.L., Kucera, T.A., Davila, J.M., St. Cyr, O.C.: 2008, Space Sci. Rev. 136, 5.

Luhmann, J.G., Curtis, D.W., Schroeder, P., McCauley, J., Lin, R.P., Larson, D.E., Bale, S.D., Sauvaud, J.-A., Aoustin, C., Mewaldt, R.A., et al.: 2008, Space Sci. Rev. 136, 117.

Maksimovic, M., Zouganelis, I., Chaufray, J.-Y., Issautier, K., Scime, E.E., Littleton, J.E., Marsch, E., McComas, D.J., Salem, C., Lin, R.P., Elliott, H.: 2005, J. Geophys. Res. 110, A09104.

Marsch, E.: 2006, Living Rev. Solar Phys. 3, 1.

McComas, D.J., Bame, S.J., Feldman, W.C., Gosling, J.T., Phillips, J.L.: 1992, Geophys. Res. Lett. 19(12), 1291. 
Opitz, A., Karrer, R., Wurz, P., Galvin, A.B., Bochsler, P., Blush, L.M., Daoudi, H., Ellis, L., Farrugia, C.J., Giammanco, C., et al.: 2009, Solar Phys. 256, 365.

Opitz, A., Fedorov, A., Wurz, P., Szego, K., Sauvaud, J.-A., Karrer, R., Galvin, A.B., Barabash, S., Ipavich, F.: 2010, Solar Phys. 264, 377.

Pilipp, W.G., Miggenrieder, H., Montgomery, M.D., Muhlhauser, K.-H., Rosenbauer, H., Schwenn, R.: 1987, J. Geophys. Res. $92(\mathrm{~A} 2), 1075$.

Richardson, J.D., Dashevskiy, F., Paularena, K.I.: 1998, J. Geophys. Res. 103(A7), 14619.

Sauvaud, J.-A., Larson, D., Aoustin, C., Curtis, D., Medale, J.-L., Fedorov, A., Rouzaud, J., Luhmann, J., Moreau, T., Schroeder, P., et al.: 2008, Space Sci. Rev. 136, 227. 